\title{
EL BARROCO LITERARIO ESPAÑOL COMO VÍA DE SOLUCIÓN DE LOS PROBLEMAS DETECTADOS EN EL GIRO SUBJETIVO DEL ÚLTIMO FOUCAULT ${ }^{1}$
}

\author{
ÓSCAR BARROSO FERNÁNDEZ \\ Universidad de Granada
}

\begin{abstract}
RESUMEN: Se analizará una aporía inherente al giro subjetivo del último Foucault causada por el sostenimiento simultáneo de dos sentidos incompatibles de la irreductibilidad del sujeto y, por lo tanto, de su posible emancipación: en unos casos es posible hacer referencia a procesos de subjetivación externos a las formas de subjetividad generadas por el poder (sentido fuerte); pero, en otros, los procesos de emancipación no pueden ser pensados más allá de la resistencia al poder (sentido débil). La hipótesis del trabajo es que la aporía está producida por la gradual marginación del problema del autoconocimiento en la temática del cuidado de sí, que, como resultado, pasa a ser comprendida exclusivamente desde las prácticas cínicas. Pero reducido el autoconocimiento, también es eliminada la problemática del cuidado de sí, que sostenía la noción fuerte de la subjetividad. Se mostrará que en el Barroco literario y neoestoico español se pueden encontrar elementos para recuperarla.
\end{abstract}

PALABRAS CLAVE: Foucault; Barroco; subjetividad; cuidado de sí; autoconocimiento; cinismo; estoicismo.

\section{Spanish Literary Baroque as a solution of some detected problems in the subjective turn of the latest Michel Foucault}

ABSTRACT: An aporia inherent to the subjective turn of the last Foucault caused by the simultaneous sustenance of two incompatible senses of the irreducibility of the subject and, therefore, of its possible emancipation will be analyzed: in some cases it is possible to refer to processes of subjectivation external to the forms of subjectivity generated by power (strong sense); but, in others, the processes of emancipation cannot be thought beyond resistance to power (weak sense). The hypothesis of the work is that the aporia is produced by the gradual marginalization of the problem of self-knowledge in the subject of self-care, which, as a result, happens to be understood exclusively from cynical practices. But when the self-knowledge it is reduced, the problem of self-care, which sustained the strong notion of subjectivity, is also eliminated. It will be shown that in the Spanish literary and neo-stoic baroque we can find elements to recover it.

KEY WORDS: Foucault; baroque; subjectivity; self-care; self-knowledge; cynicism; stoicism.

\section{INTRODUCCIÓN}

Es habitual entre los especialistas en Foucault sostener una discontinuidad ${ }^{2}$ o un giro subjetivo en su obra a partir 1980: si en el pasado había concebido al

\footnotetext{
1 Este trabajo ha sido realizado en el marco del Proyecto I+D+I «Procesos de subjetivación: biopolítica y política de la literatura. La herencia del último M. Foucault» (FFI205-64217-P), financiado por el Ministerio de Economía y Competitividad del Gobierno de España.

2 Revel, J., Foucault, un pensamiento de lo discontinuo, Amorrortu, Buenos Aires 2010, p. 17.
} 
sujeto «como el producto pasivo de las técnicas de dominación» ${ }^{3}$, a partir de este momento se refiere a una especie de irreductibilidad del yo. Pero mientras que en unas ocasiones Foucault piensa la posibilidad de emancipación de este yo irreductible a partir de formas de subjetivación alternativas a las sujeciones generadas por el poder, en otros casos, menos optimistas, sostiene que la subjetividad emancipada sólo puede darse como resistencia a las formas de sujeción. Para distinguir entre estas dos concepciones de la emancipación posible, me referiré, respectivamente, a los sentidos fuerte y débil de la subjetividad.

El problema es que estos dos sentidos de la subjetividad no pueden convivir de forma pacífica, ya que, como muestra el «sólo» subrayado, el segundo excluye al primero.

Autores como Michael Hardt y Antonio Negri han intentado dejar atrás esta aporía privilegiando el sentido fuerte del giro subjetivo, refiriéndose, por lo tanto, a la posibilidad de «una producción alternativa de subjetividad» al poder en la obra de Foucault a partir de mediados de los años setenta ${ }^{4}$. Esta interpretación tiene dos problemas fundamentales. En primer lugar, se sustenta en una ontología, la deleuzeana, que a mi juicio es incompatible con el sentido del pensamiento foucaultiano ${ }^{5}$. En segundo lugar, se trata de una lectura que, sencillamente, no tiene en cuenta la bibliografía que apoya el sentido débil del giro.

Por su parte, Frédéric Gros ha intentado salvar la aporía a través de un enfoque evolutivo: Foucault se desharía en sus últimos años de vida de las coerciones que implicaba para su filosofía una metodología crítica, posibilitando el tránsito del sentido débil al sentido fuerte de la subjetividad ${ }^{6}$. El problema de esta interpretación está en que no tiene en cuenta que Foucault sostendrá el sentido débil del giro hasta el último curso dictado en el Collège de France poco antes de su muerte.

Mi hipótesis es que no es posible salvar la ambigüedad, ya que es el resultado de una especie de callejón sin salida producido por la marginación del problema del autoconocimiento en la temática del cuidado de sí, que, como

3 Gros, F., «Situación del curso», en: Foucault, F., El gobierno de sí y los otros, Akal, Madrid 2011, p. 484.

4 Hardt, M. y Negri, A., Commonwealth, Akal, Madrid 2011, p. 71.

5 El propio Deleuze tomó nota del callejón sin salida al que parecía conducir el planteamiento de la resistencia por parte de Foucault y entendió que su ontología constituía efectivamente una respuesta: «Para mí, el estatuto de los fenómenos de resistencia no plantea problema alguno, ya que las líneas de fuga son determinaciones primeras, pues el deseo distribuye el campo social y son más bien los dispositivos de poder los que son producidos por estas distribuciones» (Deleuze, G., «Deseo y placer», en: Dos Regímenes de Locos, PreTextos, Valencia 2008 p. 126). La razón de ello está en que mientras que el poder se sitúa en el plano de organización, los fenómenos de resistencia surgen en el plano previo de consistencia (cfr., Alcalá, F. J., «Of the Processes of Subjectivation as a Subspecies of the Event: the Deleuzian Reading of the Later Foucault», en: CLCWeb: Comparative Literature and Culture, 20(4) (2018)).

6 Gros, F., o. c., p. 329. 
resultado, pasa a ser comprendida exclusivamente desde las prácticas ascéti$\operatorname{cas}^{7}$.

Intentaré demostrar que los análisis del cinismo en El coraje de la verdad constituyen el tramo final de esta reducción del autoconocimiento, pero en tales análisis también es eliminada la problemática del cuidado de sí, que era precisamente la instancia que permitía pensar la noción fuerte de la subjetividad. En esta situación, Foucault sólo podrá sostener la noción débil.

Esto significa que sólo dejando de lado el cinismo y recuperando la dimensión de autoconocimiento del cuidado, es posible superar la tensión entre ambos sentidos de subjetividad.

Creo que en el Barroco literario y neoestoico español se pueden encontrar elementos interesantes para fortalecer esta posibilidad. Se trata de una mediación que no fue explorada por el propio Foucault, pero que quiero proponer como estrategia hermenéutica.

Procederé de la siguiente forma:

En primer lugar, mostraré algunas de las referencias textuales que pueden servir de apoyo a cada uno de estos sentidos del giro subjetivo de Foucault y discutiré la estrategia de interpretación evolutiva propuesta por Gros.

En segundo lugar, intentaré demostrar que la apuesta final de Foucault por el cinismo imposibilita el desarrollo de una subjetividad en sentido fuerte.

En tercer lugar, expondré los rasgos esenciales del cuidado de sí en el Barroco español y la importancia en él del autoconocimiento. Me apoyaré en la interpretación del asunto propuesta por el hispanista Pedro Cerezo.

Por último, veré en qué sentido puede ayudar todo esto a la recuperación del sentido fuerte del giro subjetivo.

\section{Los dos SENTIDO DEL GIRO SUBJETIVO EN EL ÚlTimo FouCAULT}

Podemos encontrar apoyo textual para cada uno de los sentidos del giro subjetivo de Foucault. Mostraré solo una selección de referencias, empezando por el sentido débil.

7 Foucault define estas prácticas como «objetos de elaboración de acuerdo con procedimientos técnicos, sobre los cuales se reflexiona, y que se modifican y se perfeccionan; que se enseñan y se transmiten mediante ejemplos; que uno pone en ejecución a lo largo de su existencia, sea en ciertos momentos privilegiados y escogidos, sea de manera regular y continua; esas prácticas echan raíces en una actitud fundamental que es la preocupación por sí mismo, el cuidado de sí; y su fin es constituir un ethos, una manera de ser y de hacer, una manera de conducirse, correspondiente a ciertos principios racionales y fundadora del ejercicio de la libertad entendida como independencia; el estudio de las prácticas de sí es pues el estudio de las formas concretas, prescripciones y técnicas adoptadas por el cuidado de sí en su papel etopoyético» (Foucault, M., El coraje de la verdad. El gobierno de sí y de los otros, II, Akal, Madrid 2014, p. 306). 
En la conferencia de 1983 «¿Qué es Ilustración?», Foucault se definía, sorprendentemente, como un ilustrado. Para ello partía de la caracterización de la Aufklärung como un ethos consistente en la "crítica permanente de nuestro ser histórico ${ }^{8}$ desde una interrogación filosófica que "problematiza a la vez la relación con el presente, el modo de ser histórico y la constitución de sí mismo como sujeto autónomo»" ${ }^{9}$. Obviamente, el objetivo no era dar con elementos trascendentales, sino intentar descubrir a través del método arqueológico y con una finalidad genealógica, «los límites actuales de lo necesario»; es decir, «aquello que no es o no es ya indispensable para la constitución de nosotros mismos como sujetos autónomos» ${ }^{10}$. Se trata, por tanto, de «relanzar tan lejos y tan ampliamente como sea posible el trabajo indefinido de la libertad ${ }^{11}$. Así, concluye Foucault: «Yo caracterizaría, pues, el ethos filosófico propio de la ontología crítica de nosotros mismos como una prueba histórico-práctica de los límites que podemos franquear y, por tanto, como un trabajo nuestro sobre nosotros mismos en tanto que seres libres» ${ }^{12}$.

La posición sostenida por Foucault en este trabajo dificulta enormemente la interpretación evolutiva de su giro subjetivo mantenida por Gros. A su juicio, en el último Foucault habría una especie de superación del punto de vista de la crítica. Gros sostiene su posición comparando el tratamiento que Foucault hace de Kant y la Aufklärung en El gobierno de sí y de los otros, curso de 19821983, con el que hiciera en 1978 en «¿Qué es la crítica?», donde se situaba en la perspectiva de una «actitud crítica» que se remontaba a los comienzos de la modernidad y en oposición a la gubernamentalidad pastoral: «Formular la cuestión de la Ilustración era reencontrar la pregunta: ¿cómo no ser tan gobernado? El problema planteado era el de una 'desujeción» ${ }^{13}$. Frente a esto, en el curso de 1982-1983 la Ilustración aparecería relacionada con el decir veraz desde una interrogación diferente: «¿qué gobierno de sí debemos postular a la vez como fundamento y como límite del gobierno de los otros?» ${ }^{14}$. El problema de la interpretación evolutiva de Gros está en que, como acabamos de ver, todavía en 1983 persistía en Foucault una actitud radicalmente crítica.

Otro texto fundamental en apoyo del sentido débil de la subjetividad es «Sujeto y Poder» de 1982. Allí, Foucault escribe: «Tenemos que promover nuevas formas de subjetividad a través de esta especie de individualidad que nos ha sido impuesta por varios siglos ${ }^{15}$. A través de ella, y no fuera de ella, dado que «antes de hablar de una libertad esencial, sería mejor hablar de un

8 Foucault, M., «¿Qué es la Ilustración?», en: De La Higuera, J. (ed.), Sobre la Ilustración, Tecnos, Madrid 2003, p. 86.

9 Ibid.

$10 \quad$ Ibid., p. 88.

11 Ibid., p. 92

12 Ibid., p. 93.

13 Gros, F., o. c., p. 329.

14 Ibíd.

15 Foucault, M., «El sujeto y el poder», en: Dreyfus, H. L. y Rabinow, P., Michel Foucault: más allá del estructuralismo y la hermenéutica, Nueva Visión, Buenos Aires 2001, p. 249. 
“agonismo" "16. Sólo puede haber poder, como algo distinto de la determinación física, en el presupuesto de la libertad y sólo puede haber libertad como posibilidad de resistencia al poder.

Curiosamente Gros ha intentado defender su interpretación evolutiva del giro subjetivo afirmando que en este texto Foucault ya estaría totalmente situado en el sentido fuerte del mismo. De forma sorprendente apoya su interpretación en el mismo pasaje desde el que yo he sostenido justo lo contrario: «El problema a la vez político, ético social y filosófico que se nos plantea hoy no es tratar de liberar al individuo del Estado y sus instituciones, sino liberarnos del Estado y del tipo de individualización que se asocia a él. Debemos promover nuevas formas de subjetividad ${ }^{17}$. El problema es que Gros ha cortado la última oración del texto, donde Foucault, como acabamos de ver, continua escribiendo lo siguiente: «(...) a través de esta forma de individuación que nos ha sido impuesta por varios siglos». Es claro que la frase completa imposibilita sostener que Foucault defendía una subjetividad en sentido fuerte en este texto.

Respecto al apoyo textual del sentido fuerte, lo encontramos en los cambios de temática que se observan en los cursos del Collège de France a partir de 1979. Tras el curso de 1978-1979, el Nacimiento de la biopolítica, Foucault inicia una investigación que tiene como objetivo analizar el contexto de surgimiento del poder como gobierno, lo que le lleva, en El gobierno de los vivos, hasta las primeras comunidades monásticas del cristianismo y la forma que en ellos tienen los «actos de verdad» ${ }^{18}$, por los que los sujetos quedan atados a una verdad. Como ha visto Gros, entre los procedimientos monásticos sobresale la «obligación de decir la verdad sobre sí mismo, estructurada por la tematización de otro (...) y la muerte (puesto que por esos ejercicios se procura renunciar definitivamente a sí mismo) ${ }^{19}$. De esta forma, en este decir la verdad sobre sí mismo queda configurada una forma eficaz de sometimiento. La conexión con el biopoder es clara: "A partir de allí, y durante mucho tiempo, quedará fijado el destino del sujeto veraz en Occidente, y buscar su verdad íntima será siempre seguir obedeciendo ${ }^{20}$.

Hasta este momento Foucault no ha variado su estrategia arqueológica (estudio histórico con el objetivo de saber cómo hemos llegado a ser los individuos que somos) y genealógica (para probar su posible transgresión); pero ahora descubre algo nuevo: constata que las técnicas monásticas de producción de obediencia tenían su origen en la antigüedad griega y romana, y observa que en aquel contexto las técnicas de gobierno, tanto de sí como de los otros, no perseguían un objetivo de sometimiento o sujeción, sino, al contrario, la autonomía a través de formas de subjetivación que no podían ser reducidas a las lógicas

16 Ibíd., p. 254.

17 Gros, F., "Situación del curso», en: Foucault, M., La hermenéutica del sujeto, Akal, Madrid 2005, p. 501.

18 Foucault, M., El gobierno de los vivos, Akal, Madrid 2016, p. 88.

19 Gros, F., «Situación del curso», en: La hermenéutica del sujeto, pp. 469-470.

20 Ibid., p. 470. 
del poder ${ }^{21}$. A partir de ahora, y hasta el momento en que hace acto de aparición la parresia cínica en el curso de 1983-1984 El coraje de la verdad, Foucault dedicará sus fuerzas al estudio de las prácticas del cuidado de sí.

Creo que la toma de distancia final respecto a la problemática del cuidado de sí implicada en la inmersión en el cinismo, constituye una decisión que permite a Foucault salir del callejón sin salida al que se había visto conducido por su indecisión a la hora de elegir entre dos formas irreconciliables de comprender la emancipación ligada a los procesos de subjetivación. Y creo, también, que en el corazón de esta última decisión hay una interpretación autolimitada del cuidado de sí clásico que afecta a la posibilidad de su reapropiación presente.

\section{LAS AUTOLIMITACIONES FOUCAULTIANAS EN LA INTERPRETACIÓN DEL CUIDADO DE SÍ}

Entre los límites que definen la posible apropiación del cuidado de sí clásico por parte de Foucault, hay dos que son especialmente interesantes: la crítica irrenunciable al humanismo y el deseo de tomar distancia respecto de ciertas dinámicas de la modernidad, donde, a su juicio, había sido privilegiado el autoconocimiento, atrapado, además, en los mathémata.

Respecto a la crítica al humanismo, no podemos olvidar que aunque el intento de pensar la subjetividad en sus poderes de emancipación obligaba a Foucault a superar la radicalidad inicial de su eufórica afirmación de la «muerte del hombre ${ }^{22}$, en absoluto esto supuso un dejar atrás la crítica al humanismo. Es más, ésta aparece como una condición de posibilidad de dicha emancipación: "El humanismo sirve para embellecer y justificar las concepciones del

21 En realidad esto ya había sido descubierto por Foucault a la altura del curso del Collège de France de 1977-1978: "cuando en Grecia uno se somete a un profesor de filosofía, lo hace para llegar en determinado momento a ser maestro de sí mismo, vale decir, a trastocar esa relación de obediencia y convertirse en su propio amo. Ahora bien, en la obediencia cristiana no hay fin; en efecto ¿adónde conduce, qué es esa obediencia? Es simplemente obediencia» (Foucault, M., Seguridad. Territorio. Población, Akal, Madrid 2008, p. 177).

${ }_{22}$ Recordemos que «la muerte del hombre» no se lleva por delante sólo «las quimeras de los nuevos humanismos» (Foucault, M., Las palabras y las cosas. Una arqueología de las ciencias humanas, Siglo XXI, Buenos Aires 2006, p. 9), su ingenuidad antropocéntrica y su talante metafísico, sino que acaba también con el humanismo concebido en su acepción más general, es decir, como confianza en la emancipación sustentada en una filosofía de la subjetividad. Esto queda claro en la pasión por el sistema que Foucault manifiesta en la entrevista con Madeleine Chapsal en 1966 (Foucault, M., Dits et Écrits I, Gallimard, Paris 1994, pp. 513-518) o en la de 1968 «Foucault répond à Sartre» (Ibíd., pp. 662-668). En ellas, el "sentido" y con él, la subjetividad, aparecen como mero «effet de Surface, un miroitement, une écume, et que qui nous traversait profondément, ce qui était avant nous, ce qui nous soutenait dans le temps et l'espace, c'était le système» (Ibíd., p. 514). En referencia a Lacan, escribe Foucault: "ce sont les structures, le système même du langage —et non pas le sujet- qui parlent» (Ibíd., p. 515). En conclusión, «l'homme sujet de sa propre conscience et da sa propre liberré, c'est au fond une sorte d'image corrélative de Dieu»(Ibíd., p. 664). 
hombre a las cuales está completamente obligado a recurrir» ${ }^{23}$. En «El sujeto y el poder» (1982), este carácter justificativo del humanismo toma la forma del ejercicio efectivo del poder, el cual consiste «en guiar la posibilidad de conducta y disponerlas con el propósito de obtener posibles resultados» ${ }^{24}$. A través de la fijación de una idea de la naturaleza humana, el poder puede dirigir la conducta de los individuos y los grupos. Humanismo es, por lo tanto, técnica de gobierno, de sujeción. Y para Foucault, detrás de toda idea de humanidad podemos encontrar esta búsqueda de sujeción. Desde aquí, en normal que concluya afirmando que «hoy en día el objetivo más importante no sea descubrir qué somos sino rehusarnos a lo que somos» ${ }^{25}$.

Creo que este rechazo de la temática humanista explica en gran parte la tensión que vamos a observar desde la primera clase del curso de 1981-1982 Hermenéutica del sujeto; tensión que atraviesa toda la investigación en torno al cuidado de sí y que tiene su origen, como observa el propio Foucault, en la "cuestión de las relaciones entre la epimeleia heautou (la inquietud de sí) y el gnothi seauton (el 'conócete a ti mismo')» ${ }^{26}$.

Foucault pretende, frente a la tendencia de la filosofía moderna a privilegiar el autoconocimiento y marginar la inquietud, mostrar que el primero ocupa un lugar de subordinación respecto a la segunda ${ }^{27}$. Repite continuamente que no rechaza el autoconocimiento sin más sino sólo en su forma metafísico-platónica, pero lo cierto es que tiene graves problemas para pensar qué puede ser aquel sin esta forma. Como resultado de ello, acaba realizando un movimiento inverso al de la concepción intelectualista de la subjetividad, dejando en un segundo plano el momento de autoconocimiento, en tanto que ahora constituye «una suerte de aplicación concreta, precisa y particular, de la regla general: debes ocuparte de ti mismo» ${ }^{28}$ y concibiendo la inquietud desde las prácticas

23 Foucault, M., “¿Qué es la Ilustración?», p. 89

24 «El sujeto y el poder», en: Dreyfus, H. L. y Rabinow, P., Michel Foucault: más allá del estructuralismo y la hermenéutica, Nueva Visión, Buenos Aires 2001, p. 253.

25 «The Subject and Power», en: Critical Inquiry, 8(4) (1982), p. 785. Esta traducción no corresponde a la que se hace en la edición en español que venimos manejando de «El sujeto y el poder» y que cambia totalmente el sentido de lo que Foucault pretende decirnos: "Maybe the target nowadays is not to discover what we are, but to refuse what we are» (Ibid., p. 785).

26 Foucault, M., La hermenéutica del sujeto. Akal, Madrid 2005, p. 15.

27 Curiosamente esta idea aparecía con fuerza en el manuscrito que cerraba el curso sobre El coraje de la verdad, constituyendo por lo tanto, la última palabra de Foucault en el Collège de France sobre este asunto: "[Platón] acentúa de manera muy significativa la importancia y la amplitud de los mathémata; da al conocimiento de sí la forma de la autocontemplación y del reconocimiento ontológico de lo que es el ser propio del alma» (Foucault, M., El coraje de la verdad, p. 307). Y al mismo tiempo que censura a Platón por ello, alaba la lectura cínica de Sócrates en tanto que «reduce de la manera más rigurosa posible el ámbito de los mathémata, da al conocimiento de sí la forma privilegiada del ejercicio, la prueba las prácticas de resistencia» (Ibid). En la concepción cínica del cuidado, por lo demás totalmente inundado por la problemática de la parrhesía, el autoconocimiento parece haber sucumbido completamente ante la áskesis.

28 Foucault, M., La hermenéutica del sujeto, p. 18. 
de sí. El cuidado puede ser pensado sin el autoconocimiento y en referencia exclusiva a las prácticas.

Si Foucault se demora en el análisis de la parrhesía en el cinismo, es porque el carácter rudimentario de su antropología le sirve como excusa perfecta para marginar el autoconocimiento y, con ello, posibilitar una «estilística de la existencia $»^{29}$ desligada de todo compromiso antropológico y humanista ${ }^{30}$. Pese a las continuas precauciones para dar a entender que no se trata de una exclusión absoluta del autoconocimiento, acaba pensado, apoyándose en el cinismo, la «verdadera vida», la vida parresiástica, en contraposición a la verdad: «En este Occidente que ha inventado muchas verdades diversas y modelado artes de la existencia tan múltiples el cinismo no deja de recordar esto: que bien poca verdad es indispensable para quien quiere vivir verdaderamente, y que bien poca vida es necesaria cuando nos atenemos verdaderamente a la verdad ${ }^{31}$.

Respecto al segundo punto, no podemos olvidar que Foucault fue al cuidado de sí antiguo a la búsqueda de una forma de emancipación que permitiera superar el encierro antropológico de la subjetividad en la modernidad. En este sentido, en el prefacio de 1984 a su Historia de la sexualidad podemos leer lo siguiente: «Para mejor analizar en sí mismas las formas de la relación consigo me vi en la necesidad de remontarme en el tiempo cada vez más lejos del marco cronológico que me había fijado ${ }^{32}$.

Este cambio de interés obedece al menos a una doble razón. En primer lugar, como observa Gros reflexionando en torno a los manuscritos inéditos que sirvieron a Foucault para dictar su Hermenéutica del sujeto: «(...) lo que estructura la oposición entre el sujeto antiguo y el sujeto moderno es una relación inversa de subordinación entre inquietud de sí y autoconocimiento. (...) el sujeto de la acción recta de la antigüedad es sustituido, en el Occidente moderno, por el sujeto del conocimiento verdadero» ${ }^{33}$. En segundo lugar, es claro que para

29 Foucault, M., El coraje de la verdad, p. 153.

30 El argumento con el que deja de lado el autoconocimiento recuerda mucho la marginación del humanismo en "¿Qué es Ilustración?». Considera que una estilística de la existencia jamás puede ser entendida como «la puesta en práctica de algo parecido a una metafísica del alma», en tanto que una misma estilística puede tener tras de sí diversas metafísicas (Ibíd., p. 153). Ello quiero decir que la problemática vinculada al autoconocimiento sería demasiado flexible como para resultar fundamental para el proyecto de una estilística. A esto se une el hecho de que también una misma metafísica puede servir de justificación para diversas estilísticas. Creo que todo ello sirve para entender que Foucault está situando la problemática del autoconocimiento en la perspectiva del humanismo.

31 Ibíd., p. 177. Obviamente, Foucault era consciente de que sin la participación de discursos (logoi) verdaderos no puede haber buenas prácticas de cuidado de sí, pero concibe dichos discursos como conocimientos del mundo y no como conocimientos antropológicos: «No son en modo alguno un desciframiento de nuestros pensamientos, nuestras representaciones, nuestras presentaciones, nuestros deseos» (FoucAult, M., La hermenéutica del sujeto, p. 561).

32 Foucault, M., "El retorno de la moral», en: Estética, ética y hermeneútica, Paidós, Madrid 1999, p. 583

33 Foucault, M., La hermenéutica del sujeto, pp. 482-483. 
Foucault el autoconocimiento moderno se había visto atrapado en el callejón sin salida de los mathémata.

\section{El cinismo, un mal aliado en la defEnsa del cuidado de sí}

La relevancia concedida al cinismo en El coraje de la verdad tuvo dos consecuencias nefastas para el cuidado de sí: por un lado, como ya he apuntado, acabó anegándolo; por otro lado, hizo invisible una dimensión virtuosa del autoconocimiento moderno.

En primer lugar, por lo tanto, arruinó las posibilidades de una verdadera apropiación del cuidado de sí, obligando a Foucault a sostener su apuesta por la emancipación exclusivamente en el sentido débil de la subjetividad; es decir, el de una resistencia efectiva al poder que tiene su único posible punto de partida en las formas de sujeción del propio poder.

Esta interpretación puede resultar extraña en tanto que el propio Foucault pretendía profundizar en el cuidado de sí a través de la parrhesía, pero, a poco que se escarbe en el asunto, se observa que dicha parrhesía se configura como un práctica de sí desmesurada que acaba minando las posibilidades efectivas de formas de subjetividad autoconstituidas y, por lo tanto, encerrando el asunto de la emancipación en el camino único de la crítica. La aptitud parresiástica parece coincidir con la actitud crítica en tanto que piensa el sujeto ético desde «las relaciones de poder en que se forma $»^{34}$. Foucault llega incluso a concebir el cinismo, más allá de su concreta configuración en la antigüedad, como la forma de una crítica parresiástica que atraviesa toda la historia de la filosofía en su cara oculta ${ }^{35}$.

A mi juicio, el problema está en que no se puede pensar el cuidado de sí sin el autoconocimiento, porque, ¿cómo puede una vida cuidar de sí misma ignorando completamente su verdad? ¿Desde qué principios iba a sostener dicho cuidado?

Pero lo cierto es que si en las escuelas helenísticas no hubo una reflexión profunda en torno al problema del autoconocimiento, ello sólo puede significar que este no se vivía de forma problemática. En este sentido, creo que el carácter rudimentario y escueto de la antropología del cinismo, no obedece a

34 Foucault, M., El coraje de la verdad, p. 72.

35 Cuando los discursos revolucionario, ontológico y científico adoptan el perfil cínico de la parresia, se vuelven profundamente críticos: "El discurso revolucionario, cuando adopta la forma de una crítica de la sociedad existente, cumple el papel de discurso parresiástico. El discurso filosófico, como análisis, reflexión sobre la finitud humana, y crítica de todo lo que puede, sea en el orden del saber o en el de la moral, desborda los límites de esa finitud, representa en algún aspecto el papel de parrhesía. En lo concerniente al discurso científico, cuando se despliega - y no puede no hacerlo, en su desarrollo mismo- como crítica de los prejuicios, de los saberes existentes, de las instituciones dominantes, de las maneras de hacer actuales, tiene en verdad ese papel parresiástico» (Ibíd., p. 39). 
su desprecio, sino más bien a su asunción acrítica. Como el propio Foucault ve, para el cinismo, «uno debe conformar su vida a los preceptos que formula» ${ }^{36}$. Si en la antigüedad la inquietud se relaciona de forma privilegiada con el «ideal de establecer en el yo cierta relación de rectitud entre actos y pensamientos (hay que actuar correctamente, según principios verdaderos» ${ }^{37}$ ) y no con el conocimiento de estos principios, es por la sencilla razón de que dichos principios no se viven como algo problemáticos, sino como algo que ha de ser asumido inflexiblemente.

Es aquí donde sobresale, en segundo lugar, la virtud del autoconocimiento moderno. A Foucault se le escapó que la insistencia antropológica de la modernidad no obedecía sólo a su carácter matemático, sino también, como diría Martin Buber, a su carácter problemático ${ }^{38}$.

Foucault denunció la reducción del valor de los actos a su función cognoscitiva, pero no tuvo en cuenta que sin autoconocimiento las prácticas están descabezadas: ¿cómo esperar que una práctica fortalezca un acto recto y virtuoso si los principios sobre los que descansa no son adecuados? ¿No corremos el peligro de caer en una especie de delirio de la virtud? Un buen acto no se define solo desde el coraje parresiástico, sino también desde la sensatez.

Una estrategia interesante hubiera sido repensar el cuidado de sí antiguo desde la preponderancia antropológica moderna y, a la inversa, mediar esta modernidad desde aquel cuidado o, incluso, rastrear en la propia modernidad formas de pensar que incluyan el cuidado de sí.

\section{Precauciones a la hora de pensar a Foucault desde una antropología}

Esta es la estrategia que precisamente quiero explorar a través del Barroco español, donde la temática del cuidado de sí convive con un fortalecimiento del autoconocimiento.

36 Ibíd., p. 220. Foucault ha subrayado la diferencia entre la ascética helenística, que «no obedece a una regula (una regla) sino a una forma (....) un estilo» (Foucault, M., La hermenéutica del sujeto, p. 396), y la cristiana, en la que la vida queda sometida a una regulación estricta. Cabría preguntase si los principios en los que se basa el «estilo» cínico no implican una estricta regulación de la existencia.

37 Gros, F., «Situación del curso», en El gobierno de sí y los otros, p. 482.

38 Gros escribe: «Según el modo moderno de subjetivación, la autoconstitución como sujeto es función de una tentativa indefinida de autoconocimiento, que ya no se desvela sino por reducir la distancia entre lo que soy verdaderamente y lo que creo ser; lo que hago, los actos que realizo, sólo tienen valor en tanto me ayudan a conocerme mejor» (Ibíd., p. 482). Pero, ¿y si el desarrollo antropológico de la modernidad tiene su fuente en una experiencia de inseguridad respecto a la que la pretensión de seguridad matemática no es más que un síntoma? En este sentido escribía Buber: «Es la parquedad propia del hombre que se encuentra en una soledad de hondura desconocida hasta entonces, y su boca pronuncia con un seco patetismo la cuestión antropológica: qu'est-ce qu'un homme dans l'infini?» (BuBER, M., ¿Qué es el hombre?, Fondo de Cultura Económica, México 1949, p. 32). 
Conscientes de que esta estrategia constituye la apuesta por un modelo humanista y antropológico del cuidado de sí, y dada la reservas que el propio Foucault tenia respecto a un proceder tal, es preciso hacer una serie de observaciones y tomar ciertas precauciones.

En primer lugar, parece claro que la historia del pensamiento ha dado de sí nociones de "humanismo» que no son reductibles al humanismo retórico e ideológico que critica Foucault ${ }^{39}$. A mi juicio, el Barroco español constituye precisamente una buena muestra de ello.

En segundo lugar, la recuperación del autoconocimiento debe evitar la tentación de un sujeto fundador no condicionado en la que, a juicio de Foucault, cayeron autores como Sartre ${ }^{40}$.

En tercer lugar, es preciso evitar el esencialismo, es decir la creencia de que hay una idea de hombre definitiva que ha de ser realizada ${ }^{41}$.

En cuarto lugar, es preciso mantener el autoconocimiento en el orden de la vida, es decir, renunciar a las fugas metafísicas y la tentación de los mathemata.

Por último, se hace también necesaria una continua vigilancia respecto de las posibles conexiones de autoconocimiento y poder ${ }^{42}$.

39 Félix Duque ha desarrollado una interesante distinción paralela a esta. Se refiere a lo que he llamado «humanismo ideológico» en los términos de «humanismo pedagógico» y al humanismo propiamente filosófico como «humanismo metafísico» (cf. DuQue, F., Contra el humanismo, Adaba, Madrid 2003).

40 Como ha visto Gros, reflexionando en torno al trabajo de Foucault «A propósito de la genealogía de la ética» (en: Dits et Écrits, Gallimard, Paris 1994): «Foucault no "descubre" en 1980 la libertad nativa de un sujeto que hasta entonces presuntamente ignoraba. No podríamos sostener que, de manera repentina, abandonó los procesos sociales de normalización y los sistemas alienantes de identificación a fin de hacer surgir, en su esplendor virginal, un sujeto libre que se crea a sí mismo en el éter ahistórico de una autoconstitución pura. Lo que reprocha a Sartre es justamente haber pensado esa autocreación del sujeto auténtico, sin raíces históricas» (Gros, F., "Situación del curso», en El gobierno de sí y los otros, pp. 484485). Pero lo cierto es que Foucault tiene en su punto de mira no sólo la libertad ligada a la condición humana en Sartre, sino que también es muy crítico con las teorías que sitúan la libertad en la naturaleza humana; frente a esto, se trata de «volver a ubicarla en el campo de otras prácticas humanas, tales como la economía, la tecnología, la política, la sociología, que pueden cumplir la función de condiciones de formación, de modelos, de lugar, de aparición, etc.» (Foucault, M. у Сномsку, N., La naturaleza humana: justicia versus poder. Un debate, Katz Editores, Buenos Aires 2006, p. 43). Es decir, más allá de la crítica de la libertad incondicionada en Sartre, Foucault rechazaría cualquier intento de pensar la naturaleza y la condición humana como condición de posibilidad de la libertad.

41 Creo que en este sentido el propio Foucault puede convertirse en un buen compañero de viaje en tanto que establecía una diferencia fundamental entre la idea de «superación de sí» en el mundo antiguo y en el cristianismo (de la que el humanismo moderno no es más que una versión secular): preparación para los acontecimientos de la vida en el primer caso; superación indefinida desde un ideal de sí en el segundo (Foucault, M., La hermenéutica del sujeto, p. 302).

42 Una de las razones por las cuales Foucault insistió en la necesidad de distinguir entre las prácticas del cuidado de sí en la antigüedad y la obsesión por la verdad del sujeto que, partiendo del cristianismo, llega hasta las ciencias del espíritu y la psicología (Ibíd., p. 241), estaba en que consideraba que esta obsesión obedecía a los fines de sujeción del biopoder. 


\section{CUIDAdO DE SÍ Y AUTOCONOCIMIENTO EN EL BARROCO ESPAÑOL}

Entre los foucaultianos es habitual considerar que el humanismo queda necesariamente enredado en estos problemas ${ }^{43}$, pero a mi juicio se trata de una presuposición injustificada, en tanto que es posible dar con formas históricas del humanismo, como la propia del Barroco español, que saldrían del enredo.

Maravall ofrece una caracterización del espíritu barroco que nos pone en condiciones de entender el potencial que tiene para ayudarnos a repensar el cuidado de sí en Foucault: «El hombre del Barroco avanzan por la senda de su vivir, cargado de la necesidad problemática, y, en consecuencia, dramática, de atender a sí mismo, a los demás, a la sociedad, a las cosas. El hombre barroco es, por excelencia, el hombre "atento", dicho sea con palabra muy gracianesca. Con todo ese contorno que le rodea y cuya relación con él será decisiva, tiene que hacerse su vida y ésta resultará de la atención que ponga en ello: "Crece el camino y crece mi cuidado", dice un verso de Fernando de Herrera. En Góngora, en Lope, en Villamedina, con mucha más fuerza en Quevedo, y no menos en Gracián, la idea de "cuidado" se repite insistentemente»"

Pero si hay un pensador que ha reflexionado sobre la filosofía del Barroco español y su clave neoestoica, ese es, sin duda, Pedro Cerezo.

A su juicio, en dicha clave se expresan los elementos fundamentales del Barroco: el cuidado de sí, el desengaño, la búsqueda de retiro, la cultura del héroe. Quizás su origen podría situarse, a comienzos del siglo XVII, tras la traducción del Encheiridion de Epicteto en 1600 y aún bajo una fuerte influencia de Horacio y Erasmo, en la Epístola Moral a Fabio de Andrés Fernández de Andrada; y alcanzaría su cenit en la obra de Gracián y Quevedo ${ }^{45}$. Aunque, ciertamente, el estoicismo ya había sido recuperado en cierta medida por el Renacimiento, ahora se trata de acogerlo desde la perspectiva de la autonomía, la resistencia

43 Así, por ejemplo, Miguel Morey se refiere a un común denominador de todo humanismo que incluiría un ideal de humanidad y la promesa de su complimiento en un «hombre nuevo» (Morey, M., El hombre como argumento, Anthropos, Barcelona 1987, p. 112). Tenemos una muestra dramática de esta idea cuando a propósito de la afirmación de Chomsky de «crear una teoría social humanista basada, si es posible, en una concepción humanista y firme de la esencia humana, o de la naturaleza humana» (Foucault y Сномsку, o. c., p. 60) con el objetivo de crear una visión de sociedad futura más justa, Foucault se pregunta: «¿no se corre el riesgo de definir esta naturaleza humana que es al mismo tiempo ideal y real —y que hasta ahora fue ocultada y reprimida - en términos tomados en préstamo de nuestra sociedad, nuestra civilización, nuestra cultura?» (Ibíd., p. 62). Y, al definir la naturaleza humana, «¿No corremos el riesgo de equivocarnos?» (Ibíd., 1971, p. 63). Obviamente, este peligro solo constituye un verdadero problema si de lo que se trata es de imponer ideas; pero toda idea debe ser llevaba a la experiencia y mostrar allí su productividad.

44 Maravall, J. A., La cultura del Barroco, Ariel, Madrid 1980, p. 351.

45 Cerezo, P., «El cuidado de sí o la cultura del héroe en la Epístola moral a Fabio», en: Morales, A. J. (ed.), Actas del Congreso Internacional Andalucía Barroca, vol. IV, Consejería de Cultura de la Junta de Andalucía, Antequera 2008, p. 52. 
moral y la búsqueda de la interioridad de la conciencia, en clara oposición tanto al fideísmo como al escolasticismo.

En una época en la que se dejaba ver una profunda crisis existencial, la búsqueda interior no podía ser entendida en términos intelectualistas. Si la situación exigía un retorno al lenguaje clásico, era preciso dejar atrás el conocimiento de sí socrático-platónico, para arribar al siguiente puerto: el del cuidado de sí propio de las culturas helenística y romana. Cuidado de sí, por lo demás, inseparable de la inquietud por los otros ${ }^{46}$, como muestran los géneros claves del Barroco, la epístola y el diálogo, pero, sobre todo, y corrigiendo el segundo, la conversación.

En efecto, es importante señalar que hablamos de conversación más que de diálogo.

En el caso concreto de Gracián, la conversación deviene sabiduría como consecuencia de la asunción, en oposición al more geométrico cartesiano, de un «more lingüístico» ${ }^{47}$ que, a través de la «lógica ingeniosa», transforma la retórica en un modelo efectivo tanto para el conocimiento como para la acción.

Algunas de las características fundamentales de esta «sabiduría conversable» resultan extremadamente relevantes para pensar a Foucault.

En primer lugar, este more lingüístico no sólo rompe con el presente cartesiano, sino también con el pasado del renacimiento naturalista; tan importante en los intentos de Hardt y Negri y de Roberto Esposito a la hora de pensar una biopolítica emancipadora a partir de Foucault pero desde una clara contaminación deleuzeana ${ }^{48}$.

Así, tras la crisis barroca, Gracián tiene que mediar la tradición humanista no con la naturaleza, respecto a la cual se ha perdido la confianza, sino con la cultura, con el «artificio», sólo a través del cual el hombre puede llegar a ser «artífice de sí mismo» ${ }^{49}$. Al respecto, el arte o artificio más radical será el propio lenguaje y, en consecuencia, este «sí mismo» deja atrás las instancias solipsistas, tanto epistemológicas como morales, que están fraguando en el cartesianismo.

En conclusión, el gran teatro del mundo ya no es la naturaleza, «sino el otro orden social del afronte entre los sujetos $»^{50} \mathrm{y}$, con ello, la conversación aparece como la única forma de sabiduría sostenible, que permite salvar «al ingenio de su extravío en una creatividad alucinada y quimérica, vital y socialmente

$46 \quad$ Ibid., p. 55.

47 Cerezo, P., «Sabiduría conversable», en: Conceptos. Revista de Investigación Graciana, 3 (2006), p. 12 .

48 Barroso, O., "Potenza e virtù. L'umanesimo dopo la morte dell'uomo»", en: Gentili, D. y Stimilli, E. (eds.), Differenze italiane. Politica e filosofia: mappe e sconfinamenti, Derive Approdi, Roma 2015, pp. 226-241

49 Cerezo, P., «Sabiduría conversable», p. 14.

50 Cerezo, P., «Homo duplex: el mixto y sus dobles», en: Casanova. J. F. G. (ed.), El mundo de Baltasar Gracián. Filosofía y literatura en el Barroco, Universidad de Granada, Granada 2002, p. 413. 
infecunda o improductiva, y salva también al juicio de un rigorismo normativo, igualmente extraño al mundo concreto e histórico de la vida» ${ }^{51}$.

En segundo lugar, la conversación no se fija como objetivo alcanzar lo común universal, cosa que ocurre en el diálogo dialéctico, sino la interrelación de los diferentes puntos de vista. Tal es el trasfondo del perspectivismo graciano, donde las perspectivas no pretenden ser superadas, sino sólo entrelazadas con la intención de enriquecer el horizonte.

Esto se debe, en tercer lugar, a que la conversación está movida más por la amistad que por la verdad objetiva: «convivir no es estar de acuerdo, ni siquiera buscar un acuerdo a todo trance, como tendemos a creer hoy desde una mentalidad intelectualista, sino querer entenderse a través del juego de las diferencias ${ }^{52}$. Comunicación no de conciencias sino de existencias; es decir, no de verdades objetivas sino de verdades francas, en las que cada uno se da al otro desde la totalidad que se es.

Por último, en ningún caso esta prioridad de la parresia respecto a la verdad objetiva implica una renuncia al autoconocimiento. En la tradición barroca, aparece como una constante la idea de que la salvación sólo es posible por el conocimiento y, en especial, por el autoconocimiento o conocimiento del alma.

Ahora bien tal conocimiento no tiene nada que ver los mathémata platónicos, con un saber inmutable depositado en el alma, sino que apunta a un viaje interior cuyo punto de partida es el desengaño producido por el descubrimiento del carácter transitorio y aparente del mundo. Desengaño que, por lo demás, establece una doble relación con la libertad: «camino de liberación del falso cuidado, por el que se puede cobrar, a la postre, la libertad sobre el mundo» ${ }^{53}$. La libertad aparece así irresolublemente unidad a la verdad.

Se trata, por tanto, de un autoconocimiento radicalmente encarnado en la vida; idea que se encuentra ejemplar y bellamente comprimida en el «endecasílabo rotundo y preciso» ${ }^{54}$ que encontramos en la Epístola moral a Fabio de Andrés Fernández de Andrada: «Iguala con la vida el pensamiento» (vers. 57). Vida y pensamiento, entrañas y conciencia, se copertenecen. Se trata, concluye Cerezo, de que «ningún acto vital escape a su luz, y que su luz sea cálida y consoladora $»^{55}$.

51 Ibid.

52 Cerezo, P., «Sentido y formas del diálogo cervantino», en: Anales de la Real Academia de Ciencias Morales y Políticas, 83 (2006), p. 270.

53 Cerezo, P., «El cuidado de sí o la cultura del héroe en la Epístola moral a Fabio», p. 57.

54 Ibid., p. 60.

55 Ibíd. 


\section{Las VEnTAJas de LA INTERPRETACión BARRoca de Foucault}

Esta mínima caracterización del Barroco español, a través de la interpretación de Cerezo, es suficiente para mostrar su enorme potencialidad a la hora de pensar el sentido fuerte de la subjetividad que hemos constatado en Foucault.

En primer lugar, la sabiduría conversable graciana permite dar con una fórmula filosófica en la modernidad aclimatada al cuidado de sí clásico y con ello establecer líneas de continuidad para su apropiación en el presente ${ }^{56}$.

En segundo lugar, el Barroco implica una reelaboración del humanismo que no sólo retoma las prácticas como elementos centrales del cuidado de sí clásico ${ }^{57}$, sino que también permite su reconciliación con una forma de au-

56 Para ser justos con Foucault en este punto, hay que decir que aunque de manera global estableció una tensión radical entre el cuidado de sí en la antigüedad y el autoconocimiento en la modernidad, hay momentos en los que atisba una recuperación del cuidado de sí en la modernidad. Así, en Seguridad. Territorio. Población observa que, a medida que en el siglo XVI desaparece el cuidado vinculado al pastoreo (o se diluye en las formas de gobierno político), «reaparece una función crucial que era la función de la filosofía, digamos, en la época helenística y que, en suma, había desaparecido durante toda la Edad Media, la filosofía como respuesta a la pregunta fundamental: ¿cómo conducirse?» (Foucault, M., Seguridad. Territorio. Población, p. 222). Esta idea será retomada en La hermenéutica del sujeto, donde muestra que autores como Montaigne llevaron a cabo "una tentativa de reconstruir una estética y una ética del yo» (Foucault, M., La hermenéutica del sujeto, p. 239); aunque se trató siempre de un intento de reconstrucción fragmentaria en absoluto comparable a lo que ocurrió en la antigüedad; se trata de una especie de filosofía en la sombra, ya que, en sus líneas más visibles, la filosofía moderna se despreocupó de la vida para centrarse en la verdad bajo la inercia de una pretensión de autovalidación como ciencia (FoucAult, M., El coraje de la verdad, pp. 217-218). Pero, ¿no podemos encontrar precisamente en el Barroco español una recuperación acabada del cuidado de sí?

57 Incluso cuando Foucault descubre huellas del cuidado de sí en la modernidad, tiende a privilegiar las prácticas del cuidado respecto al autoconocimiento (Cfr. en este mismo número: Jiménez Villar, B., «Presencia y ausencia de Montaigne en la obra de Foucault», en: Pensamiento, Vol. 76, núm. 290 (2020), pp. 707-727). Pero lo cierto es que también es posible encontrar en sus últimos cursos momentos en que atisba la pertinencia del autoconocimiento. Un caso paradigmático está en la contraposición entre las formas de autoconocimiento que proponen el Alcibíades y el Laques: "ese conocimiento de sí tiene como es evidente una forma muy diferente cuando la rendición de cuentas de sí mismo se ajusta al problema del bíos (de la vida) y no al descubrimiento del alma como realidad ontológicamente distinta (Foucault, M., El coraje de la verdad, p. 150)». También en relación con esto, escribe en materiales inéditos de 1981: "Creo que hay con ello la posibilidad de hacer una historia de lo que hemos hecho y que sea al mismo tiempo un análisis de lo que somos; un análisis teórico que tenga un sentido para lo que queremos aceptar, rechazar, cambiar de nosotros mismos en nuestra actualidad. Se trata, en suma, de salir a la búsqueda de otra filosofía crítica: una filosofía que no determine las condiciones y los límites de un conocimiento del objeto sino las condiciones y posibilidades indefinidas de transformación del sujeto» (GRos, F., «Situación del curso", en: El gobierno de sí y los otro, p. 485). No se trataría tanto de abandonar las ideas de sujeto y humanidad cuanto sus imágenes trascendentales y fundacionales. Foucault no está tanto en contra de la verdad del sujeto como de que esta sea concebida como una representación separada del cuerpo. Hay verdad, pero sólo cifrada en el cuerpo, es decir, etopoyética. Aceptado esto, hay ocasiones en las que Foucault incluso apunta a la posibilidad 
toconocimiento del alma que coincide con la vida, sorteando los peligros del esencialismo y constituyéndose como una alternativa a la mathesis cartesiana.

En tercer lugar, en el Barroco, y especialmente en el caso de Gracián, el autoconocimiento del alma tiene su punto de partida en un desengaño que constituye una forma crítica de conocimiento. Es decir, permite reconciliar autoconocimiento y crítica: el primero sirve a los propósitos tanto de pensar lo que queremos ser, como de deshacernos de aquello que nos han hecho ser.

En cuarto lugar, gracias al autoconocimiento podemos dejar atrás el peligro del delirio de la virtud implicado en la asunción acrítica de los principios que han de guiar nuestros actos. Peligro a mi juicio muy real en la forma alertúrgica de la parresia propia del cinismo.

En quinto lugar, desde el Barroco podemos dar respuesta a ciertas críticas del giro subjetivo en sentido fuerte. Me refiero a críticas como la de Žižek, quien menciona el tránsito de un Foucault "perverso» y "cínico» ${ }^{58}$, que piensa la resistencia a partir de la relación circular de ley y deseo (la resistencia se reduciría al deseo generado por la prohibición) ${ }^{59}$, a otro «humanista-elitista ${ }^{60}$ y «romántico-ingenuo» ${ }^{61}$, que, tras observar que no es posible una resistencia efectiva, una verdadera emancipación, desde aquella relación circular, transita de la mera resistencia hacia la búsqueda de subjetividades alternativas a través del «uso de los placeres» y «el cuidado del yo».

Respecto a la crítica de ingenuidad, el neoestoicismo barroco nos recuerda, como ninguna otra tradición y contra del chantaje de los proyectos exclusivamente políticos de emancipación, que se puede vivir rectamente en un mundo torcido $^{62}$. Sería reductivo ver en el cuidado de sí una perspectiva ingenua, ciega a los poderes alienantes, olvidando que incluye un enfoque estratégico respecto a estos poderes, que, desde la radical asunción de la finitud, pretende buscar espacios propicios para la emancipación. Pero, en segundo lugar, y con respecto a la crítica de elitismo, esta forma de vivir no se configura como la antítesis del compromiso político, sino como su misma condición de posibilidad. Recordemos, al respecto, que Foucault consideraba que la constitución de una «ética

de superar la oposición entre prácticas y representaciones. En este sentido, escribe en los materiales inéditos de preparación de El gobierno de sí y los otros: «interrogarnos sobre lo que somos para inferir de ello lo que conviene hacer» (Ibíd., p. 497).

58 ŽıžEK, S., El espinoso sujeto. El centro ausente de la ontología moderna, Paidós, Buenos Aires 2001, pp. 267-268.

59 No hay caminos para que los individuos rearticulen los mecanismos de poder, ya que «las resistencias al poder son generadas por la misma matriz a al que parecen oponerse. En otras palabras, su concepto del "biopoder" se propone precisamente explicar el modo en que los mecanismos disciplinarios del poder pueden constituir directamente a los individuos, penetrando en los cuerpos individuales y eludiendo el nivel de la "subjetivación"» (Ibíd., p. 269).

60 ŽıžEK, S., El sublime objeto de la ideología, Siglo XXI, Madrid 2010, p. 24.

61 ŽıžEK, S., El espinoso sujeto, p. 268.

62 Cf. SAFRANSKI, R., ¿Cuánta globalización podemos soportar?, Tusquets, Barcelona 2003. 
del yo» era «una tarea urgente, fundamental, políticamente indispensable» en tanto que «no hay otro punto, primero y último, de resistencia al poder político que en la relación de sí consigo ${ }^{63}$.

Como conclusión, podemos observar que a través de la clave neoestoica es posible dejar atrás los peligros implicados en el privilegio concedido por Foucault al enfoque cínico del cuidado de sí; entre los que se incluía la paradójica disolución parresiástica del mismo cuidado y, por lo tanto, la imposibilidad de pensar de forma unitaria la emancipación concebida como crítica y como producción de formas alternativas de subjetividad.

Si es verdad, como indica Gros, que para Foucault «el individuo sujeto no surge nunca sino en la encrucijada de una técnica de dominación y una técnica de sí ${ }^{64}$, es claro que para superar las aporías cínicas de esta encrucijada podemos apoyarnos en formas de cuidado de sí como la del estoicismo; desde éste es posible comprender "que un mismo término, el de gubernamentalidad, sirva para hacer referencia a dos cosas tan diferentes, haciendo de "superficie de contacto en la que se anudan la manera de conducir a los individuos y la manera como éstos e conducen" ${ }^{\prime 65}$.

Además el neoestoicismo puede tener una ventaja sobre el estoicismo, o al menos sobre su interpretación foucaultiana. Para todos aquellos que nos enfrentamos a la obra de Foucault intentando poner en valor el cuidado de sí, pesa como una losa la confesión, la «decepción fundamental, cruel» ${ }^{66}$ que encontramos en «El retorno de la moral», donde se cataloga a toda la filosofía antigua como un "profundo error» ${ }^{67}$. Gros interpreta, con acierto, esta afirmación como el descubrimiento, por parte de Foucault, de una disyunción insuperable en el cuidado de sí en la antigüedad: o bien cae en elitismo (como ocurre habitualmente en su versión griega) o bien se configura en una ética con pretensiones universalistas (en su versión estoico-romana) ${ }^{68}$.

Es indudable que esta percepción del estoicismo es una de las razones que explican por qué Foucault acabó buscando refugio en el cinismo. De nuevo con Gros: «Es como si, frente a las aporías de una élite de la excelencia o una moral obligatoria para todos, Foucault terminada por pensar que, en el fondo, sólo puede ser legítima una ética de la provocación y el escándalo político» ${ }^{69}$.

63 Foucault, M., La hermenéutica del sujeto, p. 240.

${ }^{64}$ Gros, F., «Situación del curso», en: El gobierno de sí y los otro, p. 485.

65 Ibíd.

66 Ibíd., p. 450.

67 Foucault, M., «El retorno de la moral», p. 383.

68 Foucault, M., "À propos de la généalogie de l'éthique», p. 397. Gros muestra que Foucault ve en Grecia «una ética como estilo de existencia, y no como normatividad moral sino en términos de afirmación de una superioridad estatutaria permitida a una elite social». Frente a ello, en Roma encontramos una «liberación de la ética con respeto a condiciones sociales» en la que incluso el esclavo puede ser libre, pero "por haberse generalizado de tal modo, la ética tienden poco a poco a imponerse como norma universal» (GRos, F., "Situación del curso», en: El gobierno de sí y los otros, p. 490).

69 Ibíd., p. 491. 
He aquí expresado en su forma más dramática, el encierro final de la emancipación en la forma de la transgresión. Creo que el neoestoicismo, del que sólo hemos propuesto un breve esbozado, podría ayudar a superar este encierro.

Universidad de Granada

Óscar Barroso Fernández

Departamento de Filosofía II

obarroso@ugr.es

[Artículo aprobado para publicación en febrero de 2020] 